Rev Inv Vet Perú 2015; 26(1): 135-139

http://dx.doi.org/10.15381/rivep.v26i1.10907

\title{
Presencia de Listeria monocytogenes en Canales Porcinas en Lima, Perú
}

\author{
Presence of Listeria monocytogenes in Pig carcasses in Lima, Peru \\ Edy Waldo Barrientos H. ${ }^{1}$, Juan Raúl Lucas L. ${ }^{1,3,4}$, Daphne Ramos D. ${ }^{1}$, \\ Mónica Rebatta T. ${ }^{1}$, Teresa Arbaiza F. ${ }^{2}$
}

\section{Resumen}

\begin{abstract}
El objetivo del estudio fue determinar la presencia de Listeria monocytogenes en muestras de canales porcinas en Lima, Perú. Se muestrearon 88 superficies de canales procedentes de un centro de beneficio. Las muestras se sometieron a un preenriquecimiento en medio BLEB y enriquecimiento microbiológico en caldo PALCAM, y el patógeno se

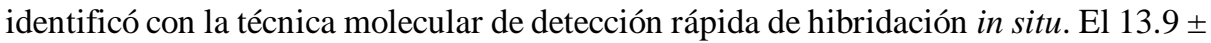
$6.1 \%$ de las canales resultaron positivas a L. monocytogenes, posiblemente debido a deficiencias en la aplicación y control de las buenas prácticas de manufactura y operaciones sanitarias estándares en el centro de beneficio.
\end{abstract}

Palabras clave: canales; centro de beneficio; cerdos; hibridación in situ

\section{Abstract}

The aim of this study was to determine the presence of Listeria monocytogenes in pig carcasses in Lima, Peru. Samples were collected from the the surface of 88 carcasses in a slaughterhouse. Samples were enriched with BLEB medium and PALCAM broth. The pathogen was identified using the in situ hybridization molecular technique. The $13.9 \pm$ $6.1 \%$ of carcasses were positive to $L$. monocytogenes, possibly due to deficiencies in the application and control of good manufacturing practices and standard sanitary operations in the slaughterhouse.

Key words: carcasses; slaughterhouse; pigs; in situ hybridization

\footnotetext{
${ }^{1}$ Laboratorio de Salud Pública y Salud Ambiental, ${ }^{2}$ Laboratorio de Bioquímica, Nutrición y Alimentación Animal, Facultad de Medicina Veterinaria, Universidad Nacional Mayor de San Marcos, Lima

${ }^{3}$ Instituto Veterinario de Investigaciones Tropicales y de Altura (IVITA, sede El Mantaro), Huancayo

${ }^{4}$ E-mail:jrlucas.pe@gmail.com
}

Recibido: 31 de marzo de 2014

Aceptado para publicación: 20 de agosto de 2014 


\section{INTRODUCCIÓN}

La bacteria intracelular Listeria monocytogenes es causante de la listeriosis en una gran variedad de especies. Este patógeno puede resistir temperaturas de congelación, infectando al hombre principalmente por la vía digestiva (Tasara y Stephan, 2006), por lo que es un agente causante de enfermedades transmitidas por alimentos (ETA).

La bacteria expresa su patogenicidad cuando enfrenta huéspedes con deficiencia de inmunidad, especialmente de tipo celular. Esta condición se observa mayormente en grupos considerados de alto riesgo, como los niños, ancianos, individuos inmunosuprimidos y mujeres gestantes (Posfay-Barbe y Wald, 2004). La listeriosis humana tiene una alta tasa de mortalidad, llegando hasta $34 \%$ (Farber y Peterkin, 1991; Pinner et al., 1992), y es en la actualidad una de las ETA de mayor importancia en el mundo.

L. monocytogenes es ubicua; sus reservorios son el medio ambiente en general, los animales y el hombre (Sammarco et al., 1997; Lida et al., 1998). Es considerada como contaminante frecuente de establecimientos de procesamiento de alimentos (centros de beneficio, salas de despiece e industrias alimentarias en general), donde es común que forme biopelículas o 'biofilms', mecanismo que la protege de condiciones adversas como cambios de temperatura y de los compuestos de lavado (Lun-den, 2004; Schöbitz et al., 2009).

El cerdo es una de las especies de producción animal de mayor consumo en el mundo. En el Perú existen más de tres millones de cerdos, se producen cerca de 115 mil toneladas métricas de carne por año, y Lima es el departamento con mayor producción (45 mil toneladas). El consumo per cápita de carne de cerdo es de $4.0 \mathrm{~kg} / \mathrm{hab} / \mathrm{año}$ a nivel nacional y de $4.3 \mathrm{~kg} / \mathrm{hab} / \mathrm{año}$ en Lima Metropolitana (MINAG, 2011).
L. monocytogenes puede aislarse en alimentos de origen animal como la carne de cerdo (Lida et al., 1998; Kanuganti et al., 2002; Rivera et al., 2006), de allí que el presente trabajo tuvo como objetivo identificar L. monocytogenes en canales porcinas resultantes del proceso de beneficio en un matadero de Lima.

\section{Materiales y Métodos}

\section{Lugar de Estudio y Muestras}

Entre mayo y junio de 2012, se colectaron muestras al azar de canales de cerdo beneficiados en un matadero autorizado de la Ciudad de Lima, Perú. Asimismo, se registraron las deficiencias observadas en las buenas prácticas de manufactura durante el proceso de beneficio (Cuadro 1).

El tamaño de muestra $(n=88)$ se obtuvo utilizando la fórmula para poblaciones infinitas (Daniel, 1996), con un nivel de confianza del $90 \%(\mathrm{~d}=0.06)$, y usando $12 \%$ como frecuencia de referencia para camales con deficiencias higiénicas (Autio et al., 2000).

En la sala de oreo se realizaron cuatro hisopados en la superficie de las canales, cada uno en un área específica (musculatura del recto abdominal y semitendinoso, y en las zonas cervical y pectoral), donde cada hisopado cubrió un área de $100 \mathrm{~cm}^{2}$. Los hisopos fueron colocados en un tubo quw contenía $10 \mathrm{ml}$ del medio BLEB (Buffered Listeria Enrichment Broth), constituyendo una sola muestra. Los tubos se transportaron en una caja térmica con hielo $\left(4-8{ }^{\circ} \mathrm{C}\right)$ al Laboratorio de Salud Pública y Salud Ambiental, Facultad de Medicina Veterinaria, Universidad Nacional Mayor de San Marcos, Lima.

\section{Detección de L. monocytogenes}

Las muestras con el BLEB se incubaron por $24 \mathrm{~h}$ a $30{ }^{\circ} \mathrm{C}$. Posteriormente se extrajo $0.1 \mathrm{ml}$ del caldo incubado, se inoculó en 
Cuadro 1. Deficiencias en las buenas prácticas de manufactura observadas durante el proceso de beneficio de porcinos en un matadero autorizado de Lima, Perú

\begin{tabular}{lll}
\hline Etapas del beneficio & Deficiencias \\
\hline $\begin{array}{l}\text { Al inicio del proceso de } \\
\text { beneficio }{ }^{1}\end{array}$ & - Ausencia del túnel de duchado previo al sacrificio. \\
$\begin{array}{l}\text { Durante el proceso de } \\
\text { beneficio }^{2}\end{array}$ & - Falta de recambio del agua del tanque de escaldado. \\
& - Operarios sin indumentaria adecuada (mascarillas, \\
& cobertores de cabello, guantes, lentes y ropa impermeable). \\
& - $\begin{array}{l}\text { Desplazamiento de los operarios desde zonas sucias } \\
\text { (escaldado o eviscerado) hacia zonas limpias (lavado). }\end{array}$ \\
& - Uso de chairas y cuchillos sucios (sin desinfectar) en la \\
& sangría y eviscerado. \\
& - Ingreso de personas ajenas al beneficio en la playa de oreo. \\
Al final del proceso de $_{\text {beneficio }^{3}}$ & Manipulación de las canales con manos sin guantes por \\
& parte del personal, vendedores y compradores. \\
\hline
\end{tabular}

En el proceso de beneficio se consideraron tres etapas: ${ }^{1}$ desde el ingreso de los animales hasta el aturdimiento; ${ }^{2}$ desde el desangrado hasta el lavado final de la canal; $\mathrm{y}^{3}$ el oreo

$10 \mathrm{ml}$ de caldo PALCAM (Polymyxin Acriflavine Lithium Chloride Ceftazidime Aesculin Mannitol) y fue incubado por $24 \mathrm{~h}$, a $35^{\circ} \mathrm{C}$.

La detección de L. monocytogenes se realizó mediante la técnica molecular de detección rápida de hibridación in situ, la cual detecta y localiza secuencias específicas de ácidos nucleícos de la bacteria. Se empleó el Kit GeneQuence ${ }^{\circledR}$ L. monocytogenes (Neogen) para el procesamiento de las muestras, siguiendo las instrucciones del fabricante (Neogen, 2009).

\section{Análisis de Datos}

Se estableció la frecuencia de canales porcinas positivas a L. monocytogenes usando la fórmula de Thrusfield (1990). Esta prevalencia se corrigió según la sensibilidad y especificidad del kit con la fórmula de Ahlborn y Norell (1990). Los resultados se expresan con un intervalo de confianza del 95\%, usando la formula de Armitage y Berry (1987).

\section{Resultados y Discusión}

Se obtuvo una frecuencia inicial de $11.4 \%$ de L. monocytogenes en las canales porcinas, que, luego de la corrección, considerando la sensibilidad (96.2\%) y especificidad $(100 \%)$ del kit, resultó en una frecuencia de $13.9 \pm 6.1 \%$. Esta frecuencia es relativamente alta, toda vez que usualmente se reporta entre 0 y $7.4 \%$ (Lida et al., 1998; Kanuganti et al., 2002; Rivera et al., 2006).

Las deficiencias durante el proceso de beneficio pueden ser la principal causa de la contaminación de las carcasas (Gahan y Collins, 1991; Sammarco et al., 1997), especialmente si se considera que $L$. monocytogenes es ubicua y forma biopelículas. Las deficiencias observadas en el matadero (Cuadro 1) explicarían en gran medida la alta frecuencia de esta bacteria en el presente estudio. Similares causantes de contaminación fueron reportados por Autio et al. (2000), donde las sierras causaban una contaminación cruzada. 
Asimismo, se sabe que las heces pueden ser fuente de contaminación de las canales (Skovgaard y Nørrung, 1989; Lida et al., 1998; Rivera et al., 2006). Además, se ha reportado una elevada frecuencia de $L$. monocytogenes en la lengua y tonsila del cerdo durante la matanza (Autio et al., 2000). Este hecho podría explicar en parte la prevalencia encontrada, dado que las carcasas de cerdo en el país se comercializan con cabeza, y esto favorecería la contaminación cruzada desde estos tejidos hacia la canal durante el procesamiento primario en el matadero.

La investigación en casos de ETA, por su repercusión en la salud pública, requiere de técnicas diagnósticas que permitan hacer un monitoreo rápido y práctico. En este contexto, los métodos de detección rápida, en especial los moleculares con enriquecimiento previo, como el método usado en el presente estudio, son bastante sensibles (Jantzen et al., 2006).

\section{Conclusiones}

Se encontró una frecuencia de $13.9 \pm$ $6.1 \%$ de Listeria monocytogenes en canales de porcinos beneficiados en un camal de Lima Metropolitana, lo cual evidencia el potencial riesgo de infección y peligro para la salud pública.

\section{Literatura Citada}

1. Ahlborn A, Norell S. 1990. Introduction to modern epidemiology. $2^{\text {nd }}$ ed. USA: Resources Inc. 273 p.

2. Armitage P, Berry G 1987. Statistical methods in medical research. $2^{\text {nd }}$ ed. UK: Blackwell Scientific. 559 p.

3. Autio T, Säteri T, FredrikssonAhomaa M, Rahkio M, Lundén J, Korkeala H. 2000. Listeria monocytogenes contamination pattern in pig slaughterhouses. J Food Prot 63: 1438-1442.
4. Daniel W. 1996. Bioestadística: base para el análisis de las ciencias de la salud. $3^{\mathrm{a}}$ ed. México: Limusa. 878 p.

5. Farber JM, Peterkin PI. 1991. Listeria monocytogenes: a food-borne pathogen. Microbiol Rev 55: 476-511.

6. Gahan GM, Collins JK. 1991. Listeriosis: biology and implications for the food industry. Trends Food Sci Tech 2: 89-93.

7. Jantzen MM, Navas J, Corujo A, Moreno $R$, López V, Martínez-Suárez JV. 2006. Specific detection of Listeria monocytogenes in foods using commercial methods: from chromogenic media to real-time PCR. Span J Agric Res 4: 235-247.

8. Kanuganti SR, Wesley IV, Reddy PG, McKean J, Hurd HS. 2002. Detection of Listeria monocytogenes in pigs and pork. J Food Prot 65: 1470-1474.

9. Lida T, Kanzaki M, Nakama A, Kokubo Y, Maruyama T, Kaneuchi C. 1998. Detection of Listeria monocytogenes in humans, animals and foods. J Vet Med Sci 60: 1341-1343.

10. Lundén J. 2004. Persistent Listeria monocytogenes contamination in food processing plants. Food Microbiol 60: 12-15.

11. [MINAG] Ministerio de Agricultura. 2011. Producción pecuaria e industria avícola 2010. Oficina de Información agraria. [Internet], [5 febrero 2014]. Disponible en: http://www2.congreso.gob.pe/sicr/ c endocbib/con 3 _ uibd.nsf/ 659B60D9CC174973052579800078A4F7/ \$FILE/2010-produccion-pecuaria.pdf

12. Neogen. 2009. GeneQuence for Listeria monocytogenes, DNA hybridization test for deteccion of listeria monocytogenes. USA: Neogen. 6 p.

13. Pinner RW, Schuchat A, Swaminathan B, Hayes PS, Deaver KA, Weaver RE, Plikaytis BD, et al. 1992. Role of foods in sporadic listeriosis. II. Microbiologic and epidemiologic investigation. The Listeria Study Group. J Am Med Assoc 267: 2046-2050. 
14. Posfay-Barbe KM, Wald ER. 2004. Listeriosis. Pediatr Rev 25: 151-159. doi: 10.1542/pir.25-5-151

15. Rivera F, Wesley I, Hurd S, Simoes D, Sosa A, Rivera S. 2006. Microbiological and molecular detection of Listeria spp and Listeria monocytogenes in a cull sows process plant in USA. Rev Cient FCV-LUZ 16: 297-307.

16. Sammarco ML, Ripabelli G, Ruberto A, Iannitto G, Grasso GM. 1997. Prevalence of Salmonellae, Listeriae, and Yersiniae in the slaughterhouse environment and on work surfaces, equipment, and workers. J Food Prot 60: 367-371.
17. Schöbitz R, Ciampi L, Nahuelquin Y. 2009. Listeria monocytogenes: un peligro latente para la industria alimentaria. Agro Sur 37(1): 1-8.

18. Skovgaard N, Norrung B. 1989. The incidence of Listeria spp in faeces of Danish pigs and in minced pork meat. Int J Food Microbiol 8: 59-63.

19. Tasara T, Stephan R. 2006. Cold stress tolerance of Listeria monocytogenes: a review of molecular adaptive mechanisms and food safety implications. $\mathbf{J}$ Food Prot 6: 1473-1484.

20. Thrusfield M. 1990. Epidemiologia veterinaria. Zaragoza: Acribia. 219 p. 